\title{
Clinical-Pathological Characteristics of Colorectal Carcinoma and Factors Influence 2-Years Survival among Patients Attending Ocean Road Cancer Institute Dar es Salaam Tanzania
}

\author{
Nebert Lulabuka $^{1^{*}(D)}$, Nazima Dharsee ${ }^{2}$, Crispin Kahesa ${ }^{2}$, Oscar Kivike ${ }^{3}$ \\ ${ }^{1}$ Departmentof Clinical Oncology, Muhimbili University of Health and Allie d Science, Dar es Salaam, Tanzania \\ ${ }^{2}$ Ocean Road Cancer Institute, Dar es Salaam, Tanzania \\ ${ }^{3}$ Department of Surgery, College of Health Sciences, The University of Dodoma, Dodoma, Tanzania \\ Email: ^lulabuka34@gmail.com,njdharsee@gmail.com,crispinkahesa@yahoo.co.uk,oscarkivike@yahoo.com
}

How to cite this paper: Lulabuka, N., Dharsee, N., Kahesa, C. and Kivike, O. (2019) Clinical-Pathological Characteristics of Colorectal Carcinoma and Factors Influence 2-Years Survival among Patients Attending Ocean Road Cancer Institute Dar es Salaam Tanzania. Open Journal of Gastroenterology, 9, 59-72.

https://doi.org/10.4236/ojgas.2019.94009

Received: December 10, 2018

Accepted: April 20, 2019

Published: April 23, 2019

Copyright $\odot 2019$ by author(s) and Scientific Research Publishing Inc. This work is licensed under the Creative Commons Attribution International License (CC BY 4.0).

http://creativecommons.org/licenses/by/4.0/

\begin{abstract}
Background: Colorectal carcinoma usually arises from an adenomatous polyp and observational studies suggest that the adenoma-to-carcinoma sequence takes approximately 10 to 15 years. Risk factors are adoption of westernized diets, obesity, cigarette smoking, and alcohol and reduced physical activity. Clinical Presentations are blood per rectum, abdominal pain, Anemia, change in bowel habits and bowel obstructive symptoms. The treatment of colorectal cancer is a dependent stage which includes chemotherapy, radiotherapy, surgery or both. Objective: This study aims at describing the clinical pathological characteristics of colorectal carcinoma and factors influencing survival among patients treated at Ocean Road Cancer Institute. Methods: This was a cross sectional study that involved histological confirmed colorectal carcinoma treated at Ocean Road Cancer Institute from 2010-2015. Results: Among 100 files extracted, 63\% were males and 37\% females. 21\% were below 40 years of age. Left-sided tumor accounted for $46 \%$. Abdominal pain, rectal bleeding and constipation were $72 \%, 68 \%$ and $55 \%$ respectively. Moderately differentiated adenocarcinoma accounted for $80 \%$. Patients presented at stage III and IV were $37 \%$ and $56 \%$ respectively. Colostomy was the most surgical procedures performed. Folinic acid, Fluorouracil and Oxaliplatin were the most common chemotherapies used. Median overall survival was estimated to be 9.4 months. Conclusion: A significant proportion of patients in this study population are young. Survival from colorectal cancer is poor owing largely to the late presentation seen in this study group.
\end{abstract}




\section{Keywords}

Colorectal Cancer Tanzania, Ocean Road Cancer Institute

\section{Introduction}

Colorectal cancer is one of the most common cancers worldwide and its incidence is reported to be increasing in resource-limited countries, probably due to the acquisition of western lifestyle [1].

The prevalence of colorectal cancer is estimated to be 1.23 million (9.7\%). There was a $1.5 \%$ decrease in incidences levels of rectal cancer and $2 \%$ increase for colon cancer every year from 2005 to 2015 [2]. Although estimates seem to suggest that the incidence is low, there is significant under-diagnosis and under-reporting.

Colorectal carcinoma usually arises from an adenomatous polyp and observational studies suggest that the adenoma-to-carcinoma sequence takes approximately 10 to 15 years. Early detection of precancerous lesions will have a major impact on colorectal cancer incidence in the population [3].

There are significant differences in the pathology of colorectal cancers according to its location within colon and rectum and therefore it is of interest to correlate symptoms of patient with tumor histology, location and TNM staging.

The risk factors of colorectal cancer are often related to adoption of westernized diets, obesity, and reduced physical activity [4].

Dietary factors account for between $30 \%$ and $50 \%$ [5]. Diet variably acts as a pro- and antitumor risk modifier across the colorectal cancer tumorigenesis process.

In Tanzania, the nutrition transitions in recent years have contributed to low fiber, high sugar, and saturated fat-ladened diets often related to processed and packaged foods. The exponential growth in availability of inexpensive calorie-ladened foods has led to gradients in energy intake between rural and urban populations [6].

Studies with diverse populations showed that physically active individuals are at a lower risk for colorectal cancer, an effect that was independent of other risk factors (i.e. diet and body weight) [7].

Cigarette smoke contains over known 60 carcinogens and free radicals, which could affect the colorectal mucosa, thereby potentiating the alteration of cancer-related genes [8].

An association between alcohol consumption and colorectal cancer was reported in more than 50 prospective and case-control studies, with no difference in the risk for colon and rectal cancers [9].

The most frequent symptoms and findings that prompted diagnostic colonoscopy in a series of 388 consecutive patients diagnosed with colorectal cancer between 2011 and 2014 included Blood per rectum 37\%, Abdominal pain 34\%, 
Anemia 23\%.

Six patients (1.9\%) had incidental colonic hyper-metabolic activity detected by PET/CT. Only four individuals (1.3\%) underwent diagnostic colonoscopy because of change in bowel habits (diarrhea). Obstructive symptoms are more common with cancers that encircle the bowel the so-called "apple-core" description seen on radiologic imaging [10]. Rectal cancer can cause tenesmus, rectal pain, and diminished caliber of stool [11].

At Ocean Road Cancer Institute the treatment of colon cancer is adjuvant chemotherapy consisting of 5-FLUROURACIL + LEUCOVORIN. Alternatively the Capecitabine can be used.

For stage IV metastatic or recurrent cancer Folinic acid, Fluorouracil and Oxaliplatin 4, and Capecitabine and Oxaliplatin are used for rectal cancer the treatment is Neoadjuvant-Chemoradiation consist of 5-FLUROURACIL + LEUCOVORIN + Radiotherapy (45 Gy) followed by Adjuvant chemotherapy.

\subsection{Problem Statement}

Colorectal ranks fourth amongst all types of cancers in East, Central South and West Africa. Also it's commonly seen at Ocean Road Cancer Institute according to the data (2006-2014) [12]. Until now the colorectal cancer survival has not been well studied in our setting, and association of clinical presentation with pathological characteristics as well as prognostic factors is not well documented.

\subsection{Broad Objective}

To describe clinical pathological characteristics of colorectal cancer and factors influence survival among patients treated at Ocean Road Cancer Institute Dar es Salaam Tanzania.

\subsection{Specific Objectives}

1) To describe social demographic characteristics of patients presenting with colorectal cancer at Ocean Road Cancer Institute Dar es Salaam Tanzania.

2) To describe clinical-pathological characteristics of patient with colorectal cancer seen at Ocean Road Cancer Institute Dar es Salaam Tanzania.

3) To describe treatment protocols received by colorectal cancer patients seen at Ocean Road Cancer Institute Dar es Salaam Tanzania.

4) To determine 2 years overall survival of colorectal cancer patients seen at Ocean Road Cancer Institute Dar es Salaam Tanzania.

5) To determine relationship between histopathological characteristics and treatment outcome of colorectal cancer patients seen at Ocean Road Cancer Institute Dar es Salaam Tanzania.

\subsection{Rationale of the Study}

This study aim was to evaluate association of clinical presentation with histological characteristics and treatment outcome of colorectal cancer patients treated 
at Ocean Road Cancer Institute. The study will help to evaluate colorectal cancer management program and address survival of patient with colorectal cancer.

\section{Methology}

\subsection{Study Design}

This is a cross-sectional study based on secondary hospital data from 2010-2015 with 2 years follow-up.

\subsection{Study Site}

The study site is Ocean Road Cancer Institute located at Dar es Salaam Tanzania. Ocean Road Cancer Institute is the only specialized facility for cancer treatment in Tanzania. The facility was established in 1895 by the German colonial government. In 1996, it was made an independent autonomous institute by an Act of Parliament. It offers patient services including laboratory services, chemotherapy, radiotherapy, palliative care and cervical cancer screening.

\subsection{Study Sample}

All files of patients who presented to Ocean Road Cancer Institute from 2010 to 2015 with histologically confirmed colorectal cancer was taken to constitute the sample.

\section{Study Variables}

\subsection{Independent Variables}

Social demographic characteristics and clinical presentation Right-sided, left-sided tumor TNM staging, histopathology, and treatment protocols.

\subsection{Dependent Variables}

Treatment outcome, HIV status and Performance status before treatment.

\subsection{Inclusion Criteria}

All patients with the histologically confirmed colon, recto-sigmoid junction and rectal cancer.

\subsection{Exclusion Criteria}

Tumors of the appendix and anal canal, carcinoid tumors, Kaposi sarcoma, lymphomas and gastrointestinal stromal tumors.

All patients with missing variables of interest.

\section{Data Collection}

Researcher assistants under the supervision of Principal investigator used data extraction forms to retrieve data from patient's records stored in patients' medical files and/or files available on the electronic medical records. 
Data extraction forms were used to collect patient's demographic data, clinical symptoms at presentation, disease stage, treatment given and laboratory and imaging report.

\subsection{Data Analysis}

All data was anonymized and analyzed using SPSS Version 23.

Data were summarized in the form of proportions and frequency tables for categorical variables. Continuous variables were summarized using mean, median mode and standard deviation. Chi-square test was used to test for significance associations between the predictor and outcome variables in the categorical variables.

\subsection{Ethical Consideration}

Ethical clearance was obtained from the Muhimbili University of Health and Allied Services Institutional Review Board and from Ocean Road Cancer Institute (ORCI) to use data from patient's records. Waiver of informed consent was obtained from the ethical committee due to the fact that the records of patients that were analyzed are available in the hospital records.

\section{Results}

\subsection{Study Population and Demographic Data}

During the study period, a total of 122 files were extracted. Of these, 22 files were excluded due to the missing information. Table 1 shows the mean age was 49.79 years with standard deviation 16.589 years. Males were $63(63 \%)$ and the number of females was $37(37 \%)$ with males to female ratio $1.7: 1$. Majority of patients $(61.0 \%)$ came from the coastal region.

Table 1. Social demographic data of the study population.

\begin{tabular}{cc}
\hline Variable & $\mathbf{n}(\%)$ \\
\hline Gender & $63(63.0)$ \\
Male & $37(37.0)$ \\
Female & $49.8(16.5)$ \\
Mean (SD) & \\
Age & $4(4.0)$ \\
Less than 20 yrs & $8(8.0)$ \\
$21-30$ & $21(21.0)$ \\
$31-40$ & $18(18.0)$ \\
$41-50$ & $49(49.0)$ \\
Above 50 & \\
Residence & $61(61.0)$ \\
I & $16(16.0)$ \\
II & $13(13.0)$ \\
III & $10(10.0)$ \\
IV &
\end{tabular}

Key: I: Coastal zone; II: Northern Highland zone: III: Lake and central zone; IV: Southern zone: the table summaries the social demographic data of patients with colorectal carcinoma seen at ORCI. 


\subsection{Anatomical Site, Gender and Stage}

Forty-one (41.0\%) patients presented with rectal carcinoma and thirty-six (36.0\%) patients diagnosed with colon carcinoma as shown in Table 2. There were twenty $(20.0 \%)$ patients diagnosed with recto-sigmoid carcinoma.

Table 3 shows that age at diagnosis was not determined the location of tumor. Males and females were both affected equally. And both patient with colon and rectal carcinoma presented at late stage (stage IV). No patients were seen during the study presented at stage I.

\subsection{Clinical Presentations and Frequency of Occurrence}

In this study, $72 \%$ of patients with CRC presented with abdominal pain, $68 \%$ presented with rectal bleeding and 55\% presented with constipation. Other symptoms were infrequently seen (Figure 1 ).

\subsection{Histological Patterns}

Microscopically, adenocarcinoma was the most common histological tumor (80.0\%) patients. (16.0\%) presented with mucinous adenocarcinoma. Squamous

Table 2. Site distribution of CRC patients.

\begin{tabular}{cccc}
\hline Site & Number $(\mathrm{n})$ & Percent $\%$ & Standard deviation (SD) \\
\hline Colon & 36 & 36.0 & 0.482 \\
Caecum & 3 & 3.0 & 0.141 \\
Recto-sigmoid & 20 & 20.0 & 0.394 \\
Rectal & 41 & 41.0 & 0.494 \\
\hline
\end{tabular}

The table summarizes the distribution of CRC among different anatomical sites. Most patients presented with colon and rectal cancers.

Table 3. Number of patients according to tumor location, gender and stages.

\begin{tabular}{ccc}
\hline Age groups & Colon & Rectal \\
\hline$<20$ & 0 & 1 \\
$21-30$ & 3 & 3 \\
$31-40$ & 7 & 10 \\
$41-50$ & 6 & 6 \\
Above 50 & 20 & 21 \\
Gender & & 27 \\
Male & 24 & 14 \\
Female & 12 & $0(0.0 \%)$ \\
Stages (TNM) & $0(0.0 \%)$ & $2(4.8 \%)$ \\
I & $2(5.6 \%)$ & $14(33.3 \%)$ \\
II & $12(33.3 \%)$ & $26(61.9 \%)$ \\
III & $22(61.1 \%)$ &
\end{tabular}

The table summarizes the number of patients diagnosed with CRC based on the age groups, gender and stage at diagnosis. 


\section{Frequency of symptoms Occurance and} distribution

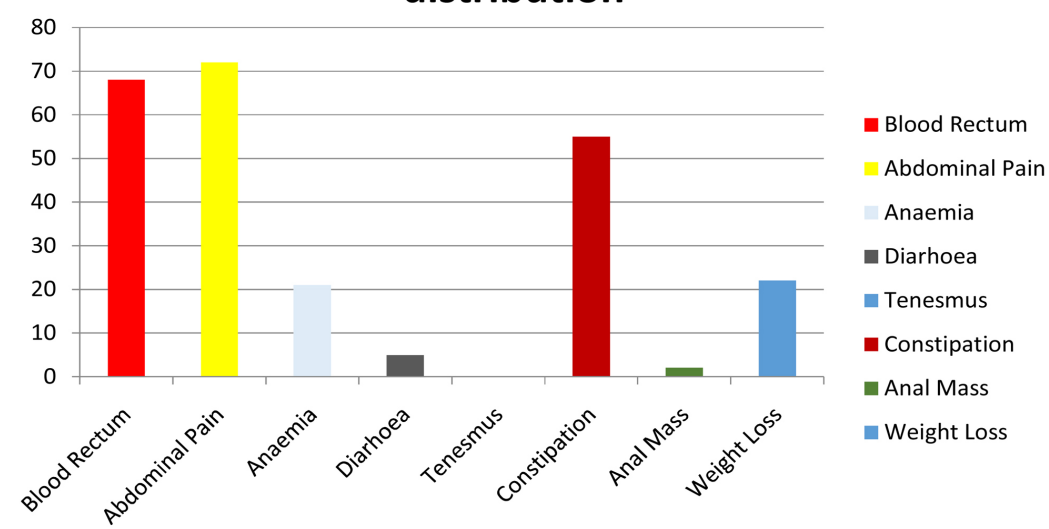

Figure 1. Distribution of patients according to clinical presentation. Abdominal pain, rectal bleeding and constipation were commonly presenting symptoms observed accounting for $72 \%, 68 \%$ and $55 \%$ respectively.

cell carcinoma was rarely seen in study (Figure 2).

The majority of the adenocarcinomas were moderately differentiated $42.0 \%$, $37.0 \%$ were well differentiated and $21.0 \%$ were poorly differentiated carcinoma (Figure 3).

According to TNM staging, most patients in this study presented at stage IV (56\%). Only $36 \%$ presented with stage III (Figure 4).

\subsection{Treatment Modalities/Regimes}

Out of 100 patients involved in this study, 43 (43.0\%) received FOLFOX4 for the treatment of colorectal carcinoma. Patients with diagnosed with rectal carcinoma were treated with RT alone as per ORCI protocols. Others were given alternative chemotherapy based on the availability.

No patients received target therapy.

Table 4 summarized the treatment received by patient with CRC treated at ORCI. Most patients received FOLFOX4.

\subsection{Response to Treatment}

Most patients with rectal cancer had no response to treatment compared to colon cancer patients but the association was not statistically significant (Table 5).

\subsection{Survival}

The patients were followed up for 2-years to asses survival of the patients with colorectal cancer, survival tends to decrease, and median overall survival was estimated to be 9.4 months (Figure 5).

Median survival time was estimated to be 9.4 months.

\subsection{Survival Rectal and Colon Carcinoma}

Survival of the patients diagnosed with colon and rectal carcinoma was performed 


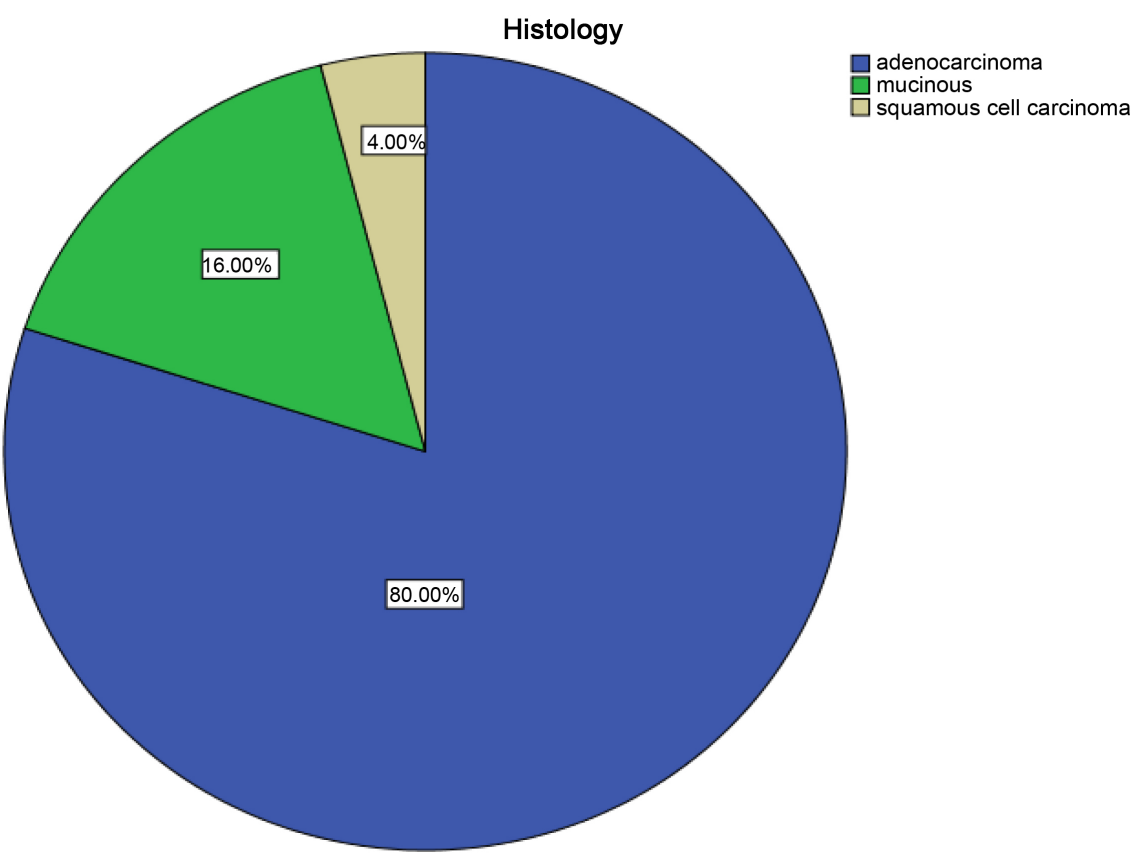

Figure 2. Represent histological pattern of colorectal carcinoma. Microscopically, adenocarcinoma was the most common histological pattern in $80 \%$ of patients. Followed by mucinous type seen in $16 \%$ of patients with colorectal carcinoma.

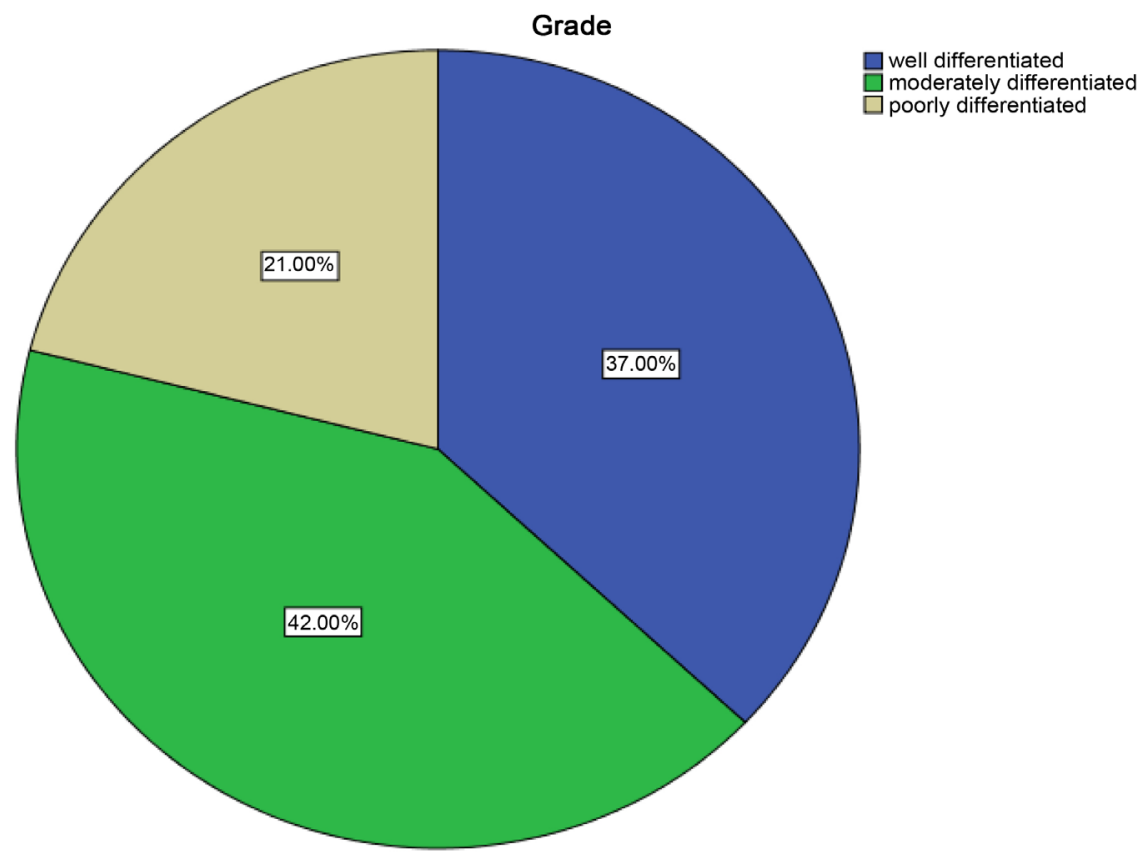

Figure 3. Degree of tumor differentiation (tumor grade) among patients with CRC. The majority of tumors were moderately differentiated which accounts for $42 \%$; well-differentiated tumor was $37 \%$, while poorly differentiated was $21 \%$.

separately (Figure 6). Colon cancer patients had slight improved survival compared to rectal cancer patients but were not statistically significant $(P=0.122)$.

According to the figure there was a trend of increased OS of patients with 


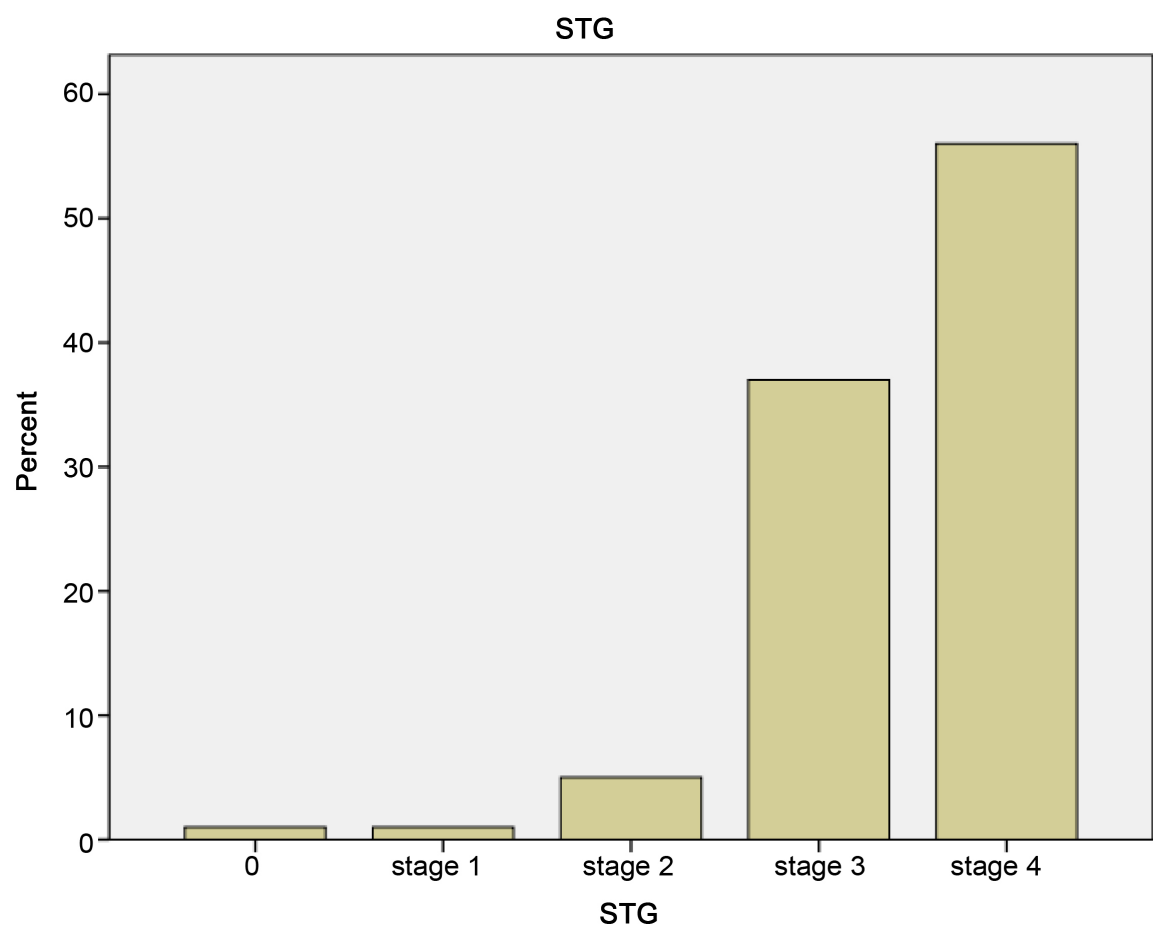

Figure 4. Tumor stage distribution.

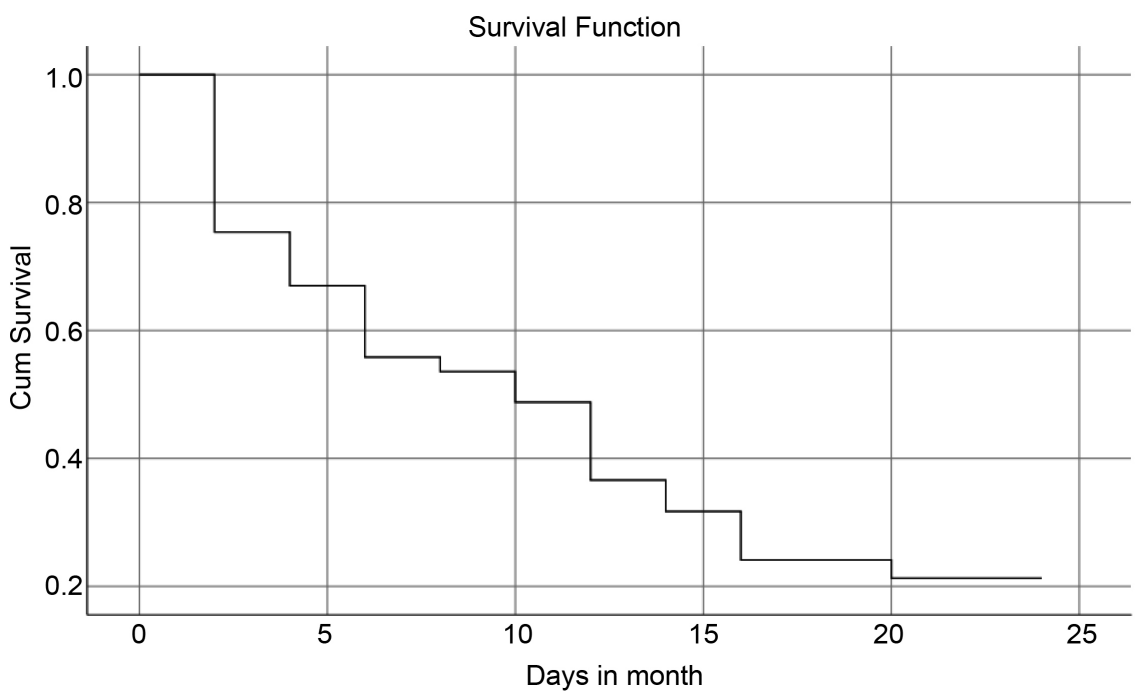

Figure 5. Overall survival of colorectal cancer patients seen at ORCI.

colon cancer compared to rectal cancer but the association was not statistically significant by log rank test.

\subsection{Predictors of Survival}

Analysis of factors associated with overall survival (OS) in colorectal carcinoma was analyzed (Table 6). The group stage (TNM) was statistically significant predictor of survival in colorectal carcinoma $(\mathrm{P}=0.003)$. Other factors were not statistically significant. 


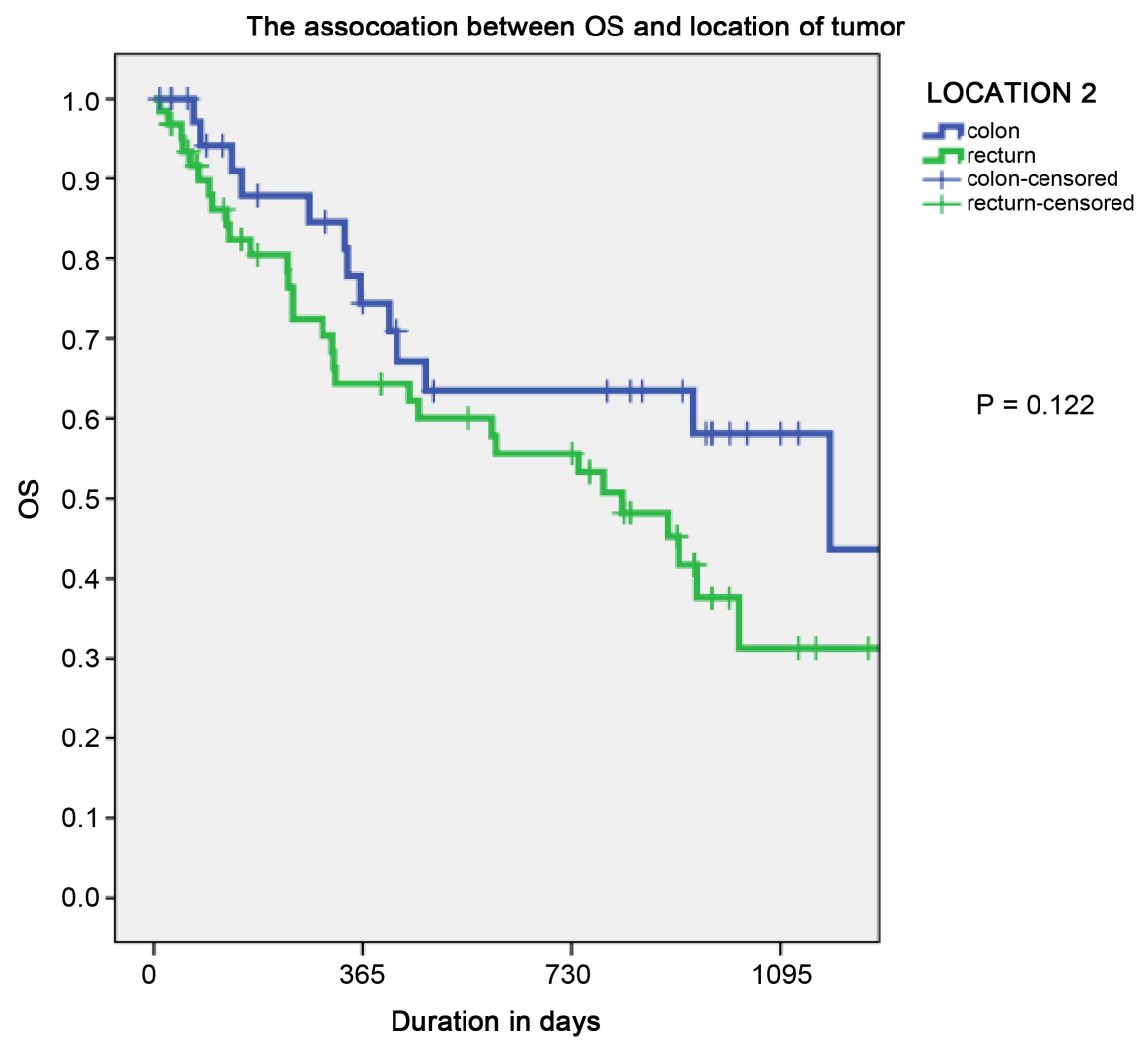

Figure 6. Overall survival of colon and rectal cancer patients.

Table 4. Treatment regimen received by patients with CRC treated at ORCI.

\begin{tabular}{ccc}
\hline Type of treatment & No. of patients & Percentage (\%) \\
\hline Chemotherapy & 43 & 43.0 \\
FOLFOX4 & 09 & 9.0 \\
CAPOX & 1 & 1.0 \\
XELOX & 12 & 12.0 \\
OTHER & 35 & 35.0 \\
RT (Rectal alone) & & \\
\hline
\end{tabular}

KEY: FOLFOX4; Oxaliplatin, Leucovorin, 5-FU. CAPOX; Capecitabine, Oxaliplatin. XELOX; Oxaliplatin, Capecitabine. RT; Radiotherapy.

Table 5. Association between tumor location and treatment response.

\begin{tabular}{cccc}
\hline & & \multicolumn{2}{c}{ Location of the tumor } \\
\cline { 3 - 4 } & & Colon & \multicolumn{1}{c}{ Rectum } \\
\hline Treatment response & CR & $8(21.1 \%)$ & $12(19.2 \%))$ \\
PR & $17(44.7 \%)$ & $15(27.3 \%)$ \\
& No response & $13(34.2 \%)$ & $35(56.5 \%)$ \\
& $38(100 \%)$ & $62(100 \%)$
\end{tabular}

Fisher's exact test 0.62 . According to the table most patients with rectal cancer had no response treatment compared to colon cancer but the association was not statistically significant. 
Table 6. The predictors of survival in study population.

\begin{tabular}{|c|c|c|}
\hline Variables & $\mathrm{N}(\%)$ & P-value \\
\hline \multicolumn{3}{|l|}{ Age } \\
\hline Below 20 & $4(4.0)$ & \multirow{4}{*}{0.341} \\
\hline $21-30$ & $8(8.0)$ & \\
\hline $31-40$ & $21(21.0)$ & \\
\hline $41-50$ & $18(18.0)$ & \\
\hline Above 50 & $49(49.0)$ & \\
\hline \multicolumn{3}{|l|}{ Histology } \\
\hline Adenocarcinoma & $80(80.0)$ & \multirow[t]{3}{*}{0.942} \\
\hline Mucinous & $16(16.0)$ & \\
\hline SCC & $4(4.0)$ & \\
\hline \multicolumn{3}{|l|}{ Residence } \\
\hline Coastal zone & $61(61.0)$ & \multirow{4}{*}{0.574} \\
\hline Northern highlands & $16(16.0)$ & \\
\hline Lake \& central & $13(13.0)$ & \\
\hline Southern zone & $10(10.0)$ & \\
\hline \multicolumn{3}{|l|}{ Group Stage (TNM) } \\
\hline I & $1(1.0)$ & \multirow{4}{*}{0.003} \\
\hline II & $5(5.0)$ & \\
\hline III & $37(37.0)$ & \\
\hline IV & $56(56.0$ & \\
\hline \multicolumn{3}{|l|}{ Grade } \\
\hline I & $36(36.0)$ & \multirow{3}{*}{0.112} \\
\hline II & $43(43.0)$ & \\
\hline III & $21(21.0)$ & \\
\hline \multicolumn{3}{|l|}{ Anatomical location } \\
\hline Left & $46(46.0)$ & \multirow{3}{*}{0.249} \\
\hline Right & $21(21.0)$ & \\
\hline Rectal & $33(33.0)$ & \\
\hline
\end{tabular}

In multiple cox regression analysis only group stage was found to be significant predictor of colorectal cancer survival with P-value of 0.003 .

\section{Discussion}

Early age onset of colorectal carcinoma was also seen in some studies [3] and the median age of onset of colorectal carcinoma was found to be 49.50 years which is three years older compared to other studies which reported median age of 46 years. In this study $21 \%$ were between age group $31-40$, the figure that is alarming. Colorectal cancer shows increase trend towards young age population, that necessitate the importance of cancer screening program and this report should be considered in future studies to evaluate predisposing and genetics factors.

Furthermore, other studies reporting age standardize incidence of 1.9 per 
100,000 in males and 1.4 per 100,000 in females [13] which is consistent to our finding which shows male predominance. Male gender have poor prognosis compared to female during analysis of this study with $\mathrm{P}=0.032$, and also males gender presented with distant metastasis $\mathrm{P}=0.031$.

The majority of patients diagnosed with CRC are males, In general, a four-to ten-year age difference by gender has been reported, with female incidence higher in the age range of $70-74$ and male incidence higher in the age range of $60-64[2]$.

Majority of patients in this study came from urban areas whereby coastal zone contributed to $60 \%$, this is different from other studies done in developing countries in which majority came from rural areas [14] [15].

The most common presenting symptoms in this study were abdominal pain. Other studies reported rectal bleeding to be the most common symptoms in these patients that prompt to seek medical advice. This may explain late presentation of CRC because these patients may be treated as other medical condition before diagnosis made.

From the study $46 \%$ of cancer occurred on the left sided while $22 \%$ were right sided tumor and $33 \%$ presented as rectal tumors. This was also reported in PROVETTA study, whereby during population study left-sided tumor was reported in $72.0 \%$ of patients [16].

Histopathologically, adenocarcinoma was the most common presentation accounting for $80.0 \%$. Similar findings were also reported in study by Chalya et al. [1].

In terms of differentiation category or tumor grade moderately differentiation (GRADE II) was reported in this study, this is contrary to WHO classification of tumor 2010 which shows $95 \%$ are well-differentiated tumors [17].

Majority of patients presented at late stage, this was also seen in some studies conducted in Africa, most of the reasons given out are lack of screening program and lack of awareness [1].

Overall survival was assessed using Kaplan-Meir and the results showed median survival was 9.4 months. This was much lower compared with other studies conducted in developed countries, which shows trends towards survival improvement in CRC patients [18].

Group stage was statistically significant factor for survival $(P=0.003)$. Study by Eetu Heerva et al. reported increase survival of colorectal carcinoma patients as compared with patients diagnosed in mid 90s. Potential factors contributing to the improvement were introduction of multidisciplinary meetings, centralization of rectal cancer surgery, and use of adjuvant chemotherapy and systemic preoperative radiotherapy of rectal cancer. In this study left-sided primary tumors had longer OS, specifically stage III disease the median OS was 7.6 years compared to 3.5 years for patient with right-sided tumor [18].

In Africa most studies reported three-year overall survival was $40 \%$, in contrast to better rates at even longer follow-up, 65\% for whites and 55\% for Africa Americans at five years [19]. 


\section{Conclusions}

$>$ The results of this study showed that colorectal carcinoma tends to occur at the age below 40 years and had low median survival of less than 1 year.

Late stage presentation was also observed in this study; many patients diagnosed with CRC at stage III and IV.

$>$ The stage of disease was the main predictor of survivorship.

From the study the common presenting symptoms were abdominal pain, rectal bleeding and constipation.

Radiotherapy seems to improve survival in colorectal cancer patient but in our study only $35 \%$ received radiotherapy; most patients received palliative dose due to resource-limited country.

\section{Recommendations}

Patients with colorectal cancer should have intensive follow-up care, if a complete colonoscopy was not possible prior to surgical resection, colonoscopy should be offered within 3 - 6 months to detect synchronous lesions.

$>$ We have identified common presenting symptoms which are key for developing tool of high index of suspicious which can be used for educating lower cadre of health professional as well as to refer early the cases.

\section{Limitations of Study}

$>$ The retrospective study design is a basic limit of the present study.

$>$ Preoperative imaging was not performed to all patients and most patients lost to follow-up.

\section{Conflicts of Interest}

The authors declare no conflicts of interest regarding the publication of this paper.

\section{References}

[1] Chalya, P.L., Mchembe, M.D., Mabula, J.B., et al. (2013) Clinicopathological Patterns and Challenges of Management of Colorectal Cancer in a Resource-Limited Setting: A Tanzanian Experience. World Journal of Surgical Oncology, 11, 1-9. https://doi.org/10.1186/1477-7819-11-88

[2] Katalambula, L.K., Ntwenya, J.E., Ngoma, T., et al. (2016) Pattern and Distribution of Colorectal Cancer in Tanzania: A Retrospective Chart Audit at Two National Hospitals. Journal of Cancer Epidemiology, 2016, Article ID: 3769829.

https://doi.org/10.1155/2016/3769829

[3] Strul, H., Kariv, R., Leshno, M., et al. (2006) The Prevalence Rate and Anatomic Location of Colorectal Adenoma and Cancer Detected by Colonoscopy in Average-Risk Individuals Aged 40-80 Years. American Journal of Gastroenterology, 101, 255-262. https://doi.org/10.1111/j.1572-0241.2006.00430.x

[4] Center, M.M., Jemal, A. and Ward, E. (2009) International Trends in Colorectal Cancer Incidence Rates. Cancer Epidemiology, Biomarkers \& Prevention, 18, 1688-1694. https://doi.org/10.1158/1055-9965.EPI-09-0090 
[5] Rothenberg, S.M. and Ellisen, L.W. (2012) The Molecular Pathogenesis of Head and Neck Squamous Cell Carcinoma. Journal of Clinical Investigation, 122, 1951-1957. https://doi.org/10.1172/JCI59889

[6] Tanner, M. and Lukmanji, Z. (1987) Food Consumption Patterns in a Rural Tanzanian Community (Kikwawila Village, Kilombero District, Morogoro Region) during Lean and Post-Harvest Season. Acta Tropica, 44, 229-244.

[7] Van Blarigan, E.L. and Meyerhardt, J.A. (2015) Role of Physical Activity and Diet after Colorectal Cancer Diagnosis. Journal of Clinical Oncology, 33, 1825-1834. https://doi.org/10.1200/JCO.2014.59.7799

[8] Parajuli, R., Bjerkaas, E., Tverdal, A., et al. (2013) The Increased Risk of Colon Cancer Due to Cigarette Smoking May Be Greater in Women than Men. Cancer Epidemiology, Biomarkers \& Prevention, 22, 862-871. https://doi.org/10.1158/1055-9965.EPI-12-1351

[9] Wang, P.-L., Xiao, F.-T., Gong, B.-C. and Liu, F.-N. (2017) Alcohol Drinking and Gastric Cancer Risk: A Meta-Analysis of Observational Studies. Oncotarget, 8, 99013-99023. https://doi.org/10.18632/oncotarget.20918

[10] Moreno, C.C., Mittal, P.K., Sullivan, P.S., et al. (2016) Colorectal Cancer Initial Diagnosis: Screening Colonoscopy, Diagnostic Colonoscopy, or Emergent Surgery, and Tumor Stage and Size at Initial Presentation. Clinical Colorectal Cancer, 15, 67-73. https://doi.org/10.1016/j.clcc.2015.07.004

[11] Popoola, A., Ibrahim, N., Omodele, F., Igwilo, I. and Soyemi, S. (2012) Clinico-Pathological Presentation of Colorectal Cancer Seen in Lagos State University Teaching Hospital. Open Access Macedonian Journal of Medical Sciences, 5, 411-415.

[12] Ocean Road Cancer Institute Cancer Registry 2006-2014.

[13] Mitry, E., Benhamiche, M., Jouve, J.L., Clinard, F., Finn-Faivre, C. and Faivre, J. (2001) Colorectal Adenocarcinoma in Patients under 45 Years of Age: Comparison with Older Patients in a Well-Defined French Population. Diseases of the Colon \& Rectum, 44, 380-387. https://doi.org/10.1007/BF02234737

[14] Abdulkareem, F.B., Abudu, E.K., Awolola, N.A., et al. (2008) Colorectal Carcinoma in Lagos and Sagamu, Southwest Nigeria: A Histopathological Review. World Journal of Gastroenterology, 14, 6531-6535. https://doi.org/10.3748/wjg.14.6531

[15] Irabor, D. and Adedeji, O.A. (2009) Colorectal Cancer in Nigeria: 40 Years on. A Review. European Journal of Cancer Care, 18, 110-115. https://doi.org/10.1111/j.1365-2354.2008.00982.x

[16] Loupakis, F., Yang, D., Yau, L., et al. (2015) Primary Tumor Location as a Prognostic Factor in Metastatic Colorectal Cancer. Journal of the National Cancer Institute, 107, 1-9. https://doi.org/10.1093/jnci/dju427

[17] Brambilla, E., Travis, W.D., Colby, T.V., Corrin, B. and Shimosato, Y. (2001) The New World Health Organization Classification of Lung Tumours. European Respiratory Society, 18, 1059-1068. https://doi.org/10.1183/09031936.01.00275301

[18] Heervä, E., Carpelan, A., Kurki, S., et al. (2017) Trends in Presentation, Treatment and Survival of 1777 Patients with Colorectal Cancer over a Decade: A Biobank Study. Acta Oncologica, 1-8.

[19] Saidi, H., Abdihakin, M., Njihia, B., et al. (2011) Clinical Outcomes of Colorectal Cancer in Kenya. The Annals of African Surgery, 7, 2009-2012. 